\title{
L'incendie créateur de quartier ou comment le risque dynamise le territoire
}

\author{
Valérie November \\ Département de géographie \\ Université de Genève \\ valerie.november@geo.unige.ch
}

\begin{abstract}
Résumé
L'incendie peut-il contribuer à créer un quartier et à jouer un rôle moteur dans la construction urbaine? Cette question prend à rebours la vision habituelle des incendies, une vision catastrophique et destructrice, où l'incendie ne laisse que cendres et débris sur son passage. Si le sinistre lui-même a des conséquences dramatiques, il peut aussi entraîner d'importantes transformations et contribuer à la création de nouvelles unités urbaines. Cependant, ce risque est rarement considéré sous cet aspect et les mécanismes procédant à la réalisation de ces transformations ont peu retenu l'attention. En s'appuyant sur un exemple (le quartier Saint-Sauveur à Québec), cet article propose une réflexion théorique sur les façons de conceptualiser l'incendie et le risque d'incendie comme faisant partie des forces qui concourent à la structuration de l'espace urbain.
\end{abstract}

Mots-clés : incendie, risques, catastrophes, territoire, dynamique urbaine, Ville de Québec, quartier Saint-Sauveur, Genève.

\begin{abstract}
Fires Creating Neighbourhoods: How Risk Transforms plans

Can fire contribute to creating a neighbourhood and play a key role in urban construction? This question flies in the face of the conventional perception of fires, which is one of catastrophe and destruction, leaving little more than ashes and debris in their wake. While the consequences of the natural disaster itself are dramatic, it can lead to major transformations and contribute to creating new urban centres. The risk of fire, however, is rarely considered in this light and the rationale behind these transformations has sparked little interest. By focusing on one example (the Quebec neighbourhood of Saint Sauveur), this article proposes a theoretical examination of the conceptualization of fire and the risk of fire as a force that contributes to the structure of urban space.
\end{abstract}

Key Words: fire, risks, catastrophes, region, urban dynamics, Québec City, community of Saint Sauveur, Geneva. 
L'incendie peut-il contribuer à créer un quartier et à jouer un rôle moteur dans la construction urbaine? Cette question prend à rebours la vision habituelle des incendies, une vision catastrophique et destructrice, où l'incendie ne laisse que cendres et débris sur son passage. Si le sinistre lui-même a des conséquences dramatiques, il peut aussi entraîner d'importantes transformations et contribuer à la création de nouvelles unités urbaines. Cependant, le risque est rarement considéré sous cet aspect et les mécanismes procédant à la réalisation de ces transformations ont peu retenu l'attention.

La capacité transformatrice, ou créatrice, de l'incendie peut être envisagée sous deux angles. Le premier consiste à débuter l'analyse en répertoriant les actions urbaines visant à effacer efficacement les traces de la catastrophe : chaque sinistre entraîne une série de reconstructions, comme dans le cas, par exemple, des villes de Londres en 1666 et de la Chaux-de-Fonds (Suisse) en 1794. La seconde perspective met l'accent sur la présence de la menace permanente que représente l'incendie en milieu urbain. Considérer simultanément ces deux angles permet alors d'envisager l'incendie comme une figure constitutive d'entités urbaines, notamment de quartiers, au sein desquels cette menace et les actions urbaines entreprises pour l'endiguer permettent à la fois de forger une identité de quartier (en positif et en négatif) et à de nouvelles formes urbaines d'émerger.

Cette double perspective oblige à une série de reformulations lors de l'analyse des incendies. En effet, elle conduit, de manière plus générale et fondamentale, à examiner la relation entre le risque d'incendie et les transformations territoriales que cette menace produit. Elle amène également à s'interroger sur les explications théoriques disponibles pour comprendre cette relation. Ainsi, cet article, tout en s'inscrivant dans la continuité des travaux sur le rôle des incendies dans l'urbanisme conduits par Jocelyne Dubois-Maury (1988a, 1988b; Chaline et Dubois-Maury, 1994 et 2002), vise à approfondir la discussion sur le rôle du risque d'incendie dans la dynamique urbaine. Pour ce faire, nous prendrons des exemples dans le quartier Saint-Sauveur, situé dans la Ville de Québec (Québec, Canada) ${ }^{1}$.

\section{RISQUES ET TERRITOIRE : ÉLÉMENTS DE DISCUSSION THÉORIQUE}

Jusqu'à récemment, les différentes formes de relation que peuvent entretenir les risques avec les territoires qu'ils affectent étaient peu explicitées. Non pas qu'il n'existe pas de travaux à ce sujet, à l'instar des études de très nombreux géographes portant sur les risques naturels ${ }^{2}$ et sur les risques technologiques majeurs ${ }^{3}$, mais ces différentes formes de relation n'ont pas fait l'objet de réflexions spécifiques. Depuis quelques années, dans le domaine des risques, on constate cependant un regain d'intérêt pour la question du territoire et, de façon intéressante, non seulement de la part des géographes, mais aussi de chercheurs issus de toutes les sciences sociales, comme des travaux de sociologues et de politologues (Decrop et al., 1997; Chateauraynaud et Torny, 1999). Cet intérêt récent des disciplines non géographiques pour le territoire révèle d'une part que la recherche en matière de risque est en pleine construction et, d'autre part, que certaines pièces du puzzle qui composent le phénomène du risque sont encore à trouver ${ }^{4}$. 
De manière plus spécifique, le milieu urbain a fait l'objet de nombreuses études en matière de risques. Ces études, dans leur majorité, ont axé leurs approches sur la notion de vulnérabilité qu'elles ont appliquées au contexte urbain ${ }^{5}$; la vulnérabilité y était comprise soit en termes probabilistes, soit en termes de représentations sociales. Ces recherches, qui concernaient essentiellement les risques naturels, n'ont toutefois pas mis l'accent sur une réflexion théorique concernant les relations entre la notion de risque et les transformations urbaines. Il en est de même pour les risques d'origine anthropique où l'intérêt s'est focalisé sur les dimensions sociales, politiques et culturelles des risques : les travaux en sciences sociales l'ont compris surtout comme un processus socioculturel (Lupton, 1999), sans accorder de signification particulière aux espaces concernés par ceux-ci.

La tendance actuelle de recherche se dirige vers une compréhension plus globale des risques et de leurs conséquences territoriales. La parution d'un volumineux ouvrage sur les risques environnementaux (Kasperson et Kasperson, 2001) ainsi qu'un autre ouvrage sur les risques urbains (Dubois-Maury et Chaline, 2002) en est le reflet, tant dans le monde anglo-saxon que francophone. L'arrivée de la notion de risque urbain n'est pas fortuite : elle résulte d'un travail commencé il y a quinze ans par des chercheurs du centre de recherche Économie et Humanisme qui plaidaient pour une conception plus générale des risques en milieu urbain. Ils interrogeaient l'approche majoritairement techniciste et probabiliste des risques (avec un fort intérêt pour les risques des réseaux techniques, domaine privilégié des ingénieurs). Ces chercheurs prônaient le passage de la notion de " risques dans le milieu urbain » à la prise en considération d'une catégorie de risques inhérents à la dynamique urbaine, à savoir les risques urbains (Lavigne et al., 1988). Ces idées ont fait lentement leur chemin, aidées par une stratégie de valorisation dans différentes revues ${ }^{6}$. Aujourd'hui, la catégorie " risques urbains » ne semble faire aucune opposition.

Curieusement, l'incendie en tant que risque urbain a été très peu étudié, a fortiori dans la perspective relationnelle que nous privilégions. À notre connaissance, seuls Jocelyne Dubois-Maury et Robert Donnell ont fait des recherches allant dans ce sens. Pour ce qui est du processus de reconstruction urbaine, nous pouvons mentionner l'ouvrage collectif dirigé par Patrick Dieudonné.

Dubois-Maury montre que la relation entre l'urbanisme et l'incendie se situe sur cinq plans complémentaires : 1) la nature des constructions (matériaux, organisation interne des locaux, disposition des accès); 2) l'état des équipements énergétiques (réseaux internes et matériel ménager); 3) l'accessibilité de l'espace urbain aux services de secours, afin de minimiser les délais d'intervention, facteur décisif de la lutte contre l'incendie : gabarit des voieries locales, accès au sein des îlots, couloirs de circulation réservés sur les axes de trafic dense; 4 ) les équipements en canalisation d'eau sous pression convenable et la densité en bornes et poteaux d'eau permettant un quadrillage satisfaisant de l'espace urbain, en fonction des potentialités d'incendie; 5) l'organisation spatiale de la ville et la distribution de ses éléments constitutifs de manière à limiter les risques de propagation du feu et à minimiser les risques en reportant en périphérie les composants urbains les plus vulnérables (Dubois-Maury, 1988b : 65-66). 
Ces cinq types de réponse à l'incendie correspondent tout à fait à ce qui peut être observé dans l'étude de cas analysée plus loin. Toutefois, ces réponses ne constituent qu'une facette de la relation entre risques et formes urbaines. Elles n'informent pas, par exemple, sur les manières dont le risque a été identifié et les modalités qui ont conduit à élaborer ces types de réponses. Elles n'informent pas, non plus, sur les différentes interactions qui existent entre ces cinq réponses, l'hypothèse étant que ce sont les différentes formes d'interactions entre ces cinq réponses au risque d'incendie qui contribuent au rôle moteur du risque dans le territoire.

Avant d'aller plus en profondeur dans l'explicitation du rôle du risque dans les transformations urbaines et d'entrer dans ce qui va servir d'illustration à notre propos théorique, il faut apporter une précision à la fois sémantique et conceptuelle sur les termes employés. Parler d'incendie nous entraîne dans deux registres connexes, mais cependant distincts, celui du risque et de la catastrophe. Nous emploierons « risque » dans un sens qui va au-delà de l'acception probabiliste, à savoir comme une menace, une possibilité qu'une situation ne se transforme en catastrophe. La catastrophe est, elle, la manifestation concrète du risque : nous considérons une catastrophe comme la "mise en visibilité » de risques sur un territoire $^{7}$. Ainsi, le risque peut être défini comme quelque chose de potentiel, qui ne s'est pas encore produit, mais dont on pressent qu'il se transformera en événement néfaste pour les individus ou pour une collectivité dans un ou des espaces donnés. Cette définition volontairement large du risque s'éloigne finalement très peu de celle qu'ont adoptée Callon, Lascoumes et Barthe dans leur étude sur les risques environnementaux. En effet, pour ces auteurs, un risque est « un danger bien identifié, associé à l'occurrence d'un événement ou d'une série d'événements, parfaitement descriptibles, dont on ne sait pas s'ils se produiront, mais dont on sait qu'ils sont susceptibles de se produire » (Callon, Lascoumes et Barthe, 2001 : 37). Seule différence notable, notre définition intègre explicitement la dimension spatiale qui se justifie pour deux motifs au moins. Quand le risque est considéré comme une projection dans le futur de signes d'une situation contemporaine qui hypothèque concrètement le territoire (en positif ou en négatif, d'ailleurs), il permet alors de faire ressortir quantité de décisions individuelles (quitter un quartier dangereux ou y rester) ou de mesures de gestion par la collectivité (zones industrielles, zones d'habitation par exemple), de dispositions réglementaires ou de politiques publiques développées à titre préventif et qui, toutes, s'inscrivent, d'une manière ou d'une autre, dans le territoire. Il s'agit de la raison « indirecte » mentionnée ci-dessus qui s'ajoute à la raison «directe » plus immédiatement intelligible. Dans cette perspective, il est important de mettre en exergue que toute identification de risques a une incidence sur le devenir des espaces qu'ils concernent.

\section{LE QUARTIER EN QUELQUES TRAITS}

\section{BRÈVE PRÉSENTATION}

La Ville de Québec est divisée en quatre secteurs, dont deux composent la ville proprement dite, la Haute-Ville et la Basse-Ville. Sur les 15 quartiers de Québec, Saint-Sauveur est l'un des quatre qui forment la Basse-Ville et est le plus densément peuplé. Ce quartier s'insère dans ce qu'on pourrait appeler une problématique de 
« Basse-Ville » : une plus grande pauvreté que dans le reste de la Ville de Québec, une tradition industrielle qui constitue aujourd'hui un héritage avec lequel il faut composer (terrains contaminés par des substances toxiques aux abords de la rivière Saint-Charles et dans le parc industriel de Saint-Malo) et qui fait obstacle à d'éventuelles constructions résidentielles sur des espaces encore vacants, un cadre environnemental plus défavorable que dans le reste de la ville (pollution de l'air et du sol). À la suite des importantes transformations du tissu métropolitain des quarante dernières années - à l'instar des métropoles nord-américaines - la population a massivement quitté les zones centrales, le taux de chômage a augmenté, l'habitat s'est dégradé, la structure commerciale et industrielle est devenue désuète. Parallèlement, de nouvelles banlieues ou de nouveaux quartiers plus éloignés, plus modernes et plus attrayants tant du point de vue fiscal que de la qualité de vie se sont développés. Ainsi, à Saint-Sauveur, la population est passée de 40000 habitants en 1940 à 19300 en 1986 et a encore diminué de 8,5\% de 1986 à 1991. Cinq ans plus tard, d'après le recensement de 1996, le quartier comptait 16189 âmes, pour une population de 167264 personnes (Ville de Québec entière). Le quartier est confronté à la fermeture d'écoles, à de mauvaises conditions socio-sanitaires ${ }^{8}$, à la détérioration du cadre bâti dans certaines parties. En 1993, Saint-Sauveur était intégré aux « zones défavorisées de la Communauté urbaine de Québec » (Bélanger, 1993 : 2).

Depuis la fin des années soixante, l'avenir des quartiers centraux demeure une préoccupation importante et très peu d'interventions urbaines ont réussi jusqu'à présent à modifier les tendances évoquées ci-dessus ${ }^{9}$. C'est dans ce contexte d'interrogations qu'ont été mises sur pied d'importantes études par la Ville de Québec. Leur mandat était de cerner la problématique des quartiers de la Basse-Ville, dont celle de Saint-Sauveur ${ }^{10}$. Ces recherches avaient pour objectif, entre autres, de réunir des informations sur l'évolution des centres d'emplois, les conditions de vie des habitants, ainsi que les fonctions résidentielles, commerciales et industrielles. Ces informations étaient en effet totalement lacunaires pour la Basse-Ville de Québec jusqu'au début des années 1990. Dix ans plus tard, une deuxième vague d'études, à dominante urbanistique et architecturale, a été lancée récemment, à l'instar des travaux réalisés par Luc Noppen et Lucie K. Morisset en 2000. L'idée était d'accumuler des connaissances spécifiques sur ce quartier, de recenser le patrimoine ainsi que les formes urbaines à valoriser.

\section{MÉTHODOLOGIE}

Pour montrer la puissance transformatrice de l'incendie, telle qu'elle se présente dans le quartier Saint-Sauveur, deux moments caractéristiques de l'histoire de ce quartier ont été choisis. Le premier concerne la création administrative du quartier Saint-Sauveur, en 1889. Le second porte sur le rôle que joue l'incendie dans l'identité du quartier au cours de la période contemporaine. Cette époque correspond en effet à une prise de conscience de l'existence d'une problématique spécifique au quartier Saint-Sauveur. Nous verrons que, finalement, la création administrative et la création en termes identitaires du quartier Saint-Sauveur reposent sur des processus similaires, où la menace d'incendie joue un rôle important. L'étude dont cet article constitue le prolongement portait sur plusieurs catégories de risques $\left(\right.$ November, 1994) ${ }^{11}$. Nous en avons extrait l'exemple du risque d'incendie, car il illustre bien la question du rôle du risque dans les transformations urbaines. C'est 
pourquoi l'exemple qui suit a valeur d'illustration : il faut l'envisager comme une discussion qui pourrait être élargie à d'autres catégories de risques, ainsi qu'à d'autres séquences temporelles ${ }^{12}$.

Les sources d'information utilisées dans cette étude sont de deux ordres. Premièrement, les documents écrits constituent la majeure partie de la récolte d'information. Ils émanent notamment des services municipaux de la Ville de Québec (urbanisme, santé, police, protection contre l'incendie) ou d'organismes travaillant à l'échelle régionale ${ }^{13}$. Des dossiers élaborés par les associations de citoyens, des ouvrages d'historiens ont été consultés. Nous avons également eu à notre disposition une base de données sur les conflits de localisation dans la ville de Québec. Celle-ci était constituée d'articles de journaux du quotidien Le Soleil (Côté, 1991).

Deuxièmement, des entretiens oraux complètent les informations écrites. Ils ont été réalisés avec des personnes travaillant en relation avec le quartier Saint-Sauveur. Certaines étaient engagées activement dans la vie du quartier - en tant que membres de comités de citoyens, de groupes d'alphabétisation - ou y travaillant - dans un centre de loisirs ou encore dans un organisme à but non lucratif (Association des Gens d'Affaires de Saint-Vallier-Ouest, par exemple), etc. D'autres étaient employées par les instances et organismes auprès de qui nous avons trouvé les informations écrites. Les entretiens réalisés avec ces dernières - travaillant au service d'Urbanisme, de Protection contre l'incendie, de Police, etc. - ont permis d'actualiser les informations écrites. Nous avons délibérément interrogé des personnes concernées par le quartier, que cela soit en raison de leur activité (bénévole) personnelle ou professionnelle, en tant qu'intervenants du milieu urbain ou parce qu'elles correspondaient au profil requis par la méthodologie. En ce sens, ces entretiens reflètent des visions qui se prononcent sur le quartier, qui émettent un jugement, même si celui-ci est contradictoire selon sa provenance (municipalité ou association communautaire, par exemple).

\section{QUEL RÔLE POUR L'INCENDIE DANS LE QUARTIER SAINT-SAUVEUR?}

\section{LA PÉRIODE DE 1889}

L'incendie est étroitement associé à l'histoire même du quartier : un grand sinistre dans le quartier voisin de Saint-Roch, en 1845, aurait été le catalyseur du développement de Saint-Sauveur. En effet, au tournant du XIXe siècle, l'essor économique provoqué à Québec par le succès des chantiers navals, du commerce du bois et de l'activité portuaire entraîne un développement fulgurant du faubourg Saint-Roch, et les nouveaux arrivants doivent s'installer désormais dans SaintSauveur, considéré comme la banlieue de Québec:

L'incendie qui détruit le quartier Saint-Roch en 1845 va devenir le catalyseur de ce débordement. En effet, le fléau jette sur le pavé un grand nombre d'ouvriers, dont certains sont très dépourvus. Ceux-ci n'ont pas les moyens financiers pour reconstruire en matériaux incombustibles, comme l'exige le nouveau règlement. 
L'idée de reconstruire à l'extérieur des limites de la ville de Québec pour se soustraire au règlement en séduit plusieurs, d'autant plus qu'en même temps, ils évitent de payer les taxes municipales (Ville de Québec, 1987 : 9).

Ainsi, une partie de la population décide d'investir cet espace hors des limites municipales de la ville de Québec.

La catastrophe entraîne parmi les grands propriétaires fonciers de l'époque (Pierre Boisseau, l'Hôpital Général, l'Hôtel-Dieu et les Ursulines) la décision de lotir rapidement. Mais les maisons qui s'élèvent alors sur les terrains pour la plupart marécageux sont modestes, voire, selon les chroniqueurs, misérables: «Boisseauville, qui fut longtemps le noyau urbain le plus important de l'actuel quartier Saint-Sauveur, était surnommé le Faubourg des tuyaux, car beaucoup d'habitations n'avaient pas de cheminées de brique, mais de simples tuyaux de tôle qui dépassaient du toit »(Ville de Québec, 1987 : 11).

Ces amas de tôles et de bois que sont les maisons de l'époque ainsi que l'augmentation de la population urbaine en dehors des limites de la ville inquiètent le maire d'alors, M. Belleau (Hare et al., $1987: 287$ et 289). À la suite des graves sinistres de 1845, le Conseil de ville réglemente la même année l'usage du bois comme matériau de construction : tous les bâtiments en bois doivent recevoir un crépi de plâtre et toute nouvelle construction où l'on fait du feu doit avoir des murs en pierre, en brique ou en d'autres matériaux incombustibles. Toutefois, il permet la reconstruction de bâtisses temporaires en bois, comme le soulignent Hare, Lafrance et Ruddel (1987 : 232) : « Ce règlement explique sans doute pourquoi les faubourgs incendiés ont pu renaître en quelques mois et être la proie des flammes régulièrement ». Le service d'Aqueduc de la Ville de Québec, inauguré en 1854, et le remplacement des pompiers volontaires par un service permanent en 1858, peuvent faire croire aux citoyens de Québec que les pires dangers sont écartés. Durant la décennie 1851-1861, malgré un retour relatif de prospérité, plus de la moitié des maisons sont toujours en bois, surtout dans les faubourgs et dans la banlieue qui ne font pas partie de la municipalité de Québec et qui ne sont par conséquent pas desservis par le réseau de distribution d'eau. Et, comme l'observent Hare, Lafrance et Ruddel (1987 : 289), la décennie suivante est marquée par un retour des grands sinistres qui dévastent surtout les quartiers ouvriers.

En 1866 éclate un immense incendie qui ravage le quartier presque en entier. Dans la désolation qui succède à la catastrophe, un changement notable peut être constaté : Saint-Sauveur obtient l'autorisation d'ériger sa propre paroisse - alors qu'elle n'était qu'une desserte de la Fabrique de Saint-Roch (Ville de Québec 1987 : 14). Le détail est capital quand on sait que la vie d'un quartier tournait alors exclusivement autour de la paroisse et que, lorsqu'un incendie survenait, les premières bâtisses reconstruites étaient l'église et l'école. Cet incendie entraîne une deuxième vague de spéculation où les Ursulines décident d'exploiter et de vendre des lotissements plus à l'ouest du quartier.

À la suite du nouveau code municipal de 1855 qui obligeait les petites villes et villages du Québec à se donner une administration municipale, Saint-Sauveur se regroupe d'abord avec Limoilou en une municipalité appelée "banlieue de Saint-Roch » jusqu'en 1862, puis obtient une charte municipale autonome en 1862, 
pour, finalement, changer son nom officiel de Saint-Roch nord en Saint-Sauveur en 1872 (Lemoine, 1982 : 6). À cette date, le quartier qui s'étend du boulevard Langelier à la rue Marie-de-l'Incarnation devient indépendant, malgré le désir exprimé régulièrement depuis 1853 par les autorités municipales de la Ville de Québec d'annexer ce territoire. En effet, selon le document du service d'urbanisme, " ce cheminement vers l'autonomie développe un sentiment d'appartenance chez les résidants de Saint-Sauveur, qui résistent à toutes les tentatives d'annexion faites par la Ville de Québec, désireuse d'agrandir son territoire et d'augmenter le nombre de ses contribuables » (Ville de Québec, 1987 : 16).

Une action urbaine est entreprise pour endiguer le feu : le boulevard Langelier est élargi après l'incendie de 1866. On désire ainsi aménager un espace coupe-feu entre Saint-Roch et Saint-Sauveur. En 1885, on le divise en deux voies séparées par un terre-plein sur lequel on plante une double rangée d'arbres (Ville de Québec, 1987 : 19). Pourtant, le quartier est à nouveau en proie aux flammes le 16 mai 1889. Comme le remarque Lemoine (1982: 6), « les citoyens paient cher le fait d'avoir rejeté par référendum en 1887 un règlement d'emprunt visant à construire un aqueduc ». L'incendie détruit le tiers de la paroisse, soit environ cinq cents maisons, et laisse quatre à cinq mille familles sans abri. Cette catastrophe relance le débat sur l'annexion avec plus d'intensité encore. Comment faire face à ce risque que constitue la menace de l'incendie? La discussion tourne autour de la distribution de l'eau et du réseau à mettre sur pied pour combattre le feu. Dès le mois de juin, des pourparlers commencent entre les deux municipalités et les citoyens de Saint-Sauveur entérinent l'entente par référendum les 26 et 27 septembre 1889 (Ville de Québec, 1987 : 18). Les modalités de l'annexion concernent notamment l'installation du réseau de distribution d'eau dans les plus brefs délais, donc des aqueducs introduits par les autorités de Québec dans les principales rues de Saint-Sauveur, la construction d'égouts, de trottoirs, le pavage et l'éclairage des rues. L'aménagement d'un parc est également une condition à l'acceptation de l'annexion par les citoyens de Saint-Sauveur (figure 1).

Ainsi, la menace de l'incendie, d'une acuité sans précédent après le sinistre de 1889, entraîne l'annexion de Saint-Sauveur par la Ville de Québec grâce à la promesse de son développement rapide. Les transformations sont spectaculaires, selon l'ingénieur de l'époque de la Ville de Québec, Charles Baillargé, puisque « en moins de trois ans, le quartier rattrape son retard sur le reste de la ville quant à l'installation des équipements urbains : réseau de distribution d'eau, réseau de drainage, trottoirs, chaussées empierrées, postes de pompiers et de police, téléphone et éclairage électrique » (Ville de Québec, 1987 : 19).

\section{L'INCENDIE : UNE MENACE PRÉSENTE ENCORE AUJOURD'HUI}

Lors de l'étude menée auprès des personnes concernées par les risques dans le quartier Saint-Sauveur ${ }^{14}$, un des risques mentionnés par tous les acteurs est la menace d'incendie. Les résidants du quartier craignent fortement, par exemple, les maisons de chambres ${ }^{15}$, ainsi que les bâtiments vacants mal placardés ${ }^{16}$. En réponse à ces inquiétudes, le Comité de citoyens et de citoyennes du quartier Saint-Sauveur a dressé au milieu des années 1970 un inventaire des maisons abandonnées qui représentaient un danger d'incendie pour les résidants du quartier, afin d'identifier les lieux du risque (figure 2). 
Figure 1 Ville de Québec, annexions successives au territoire municipal, 1833-1930

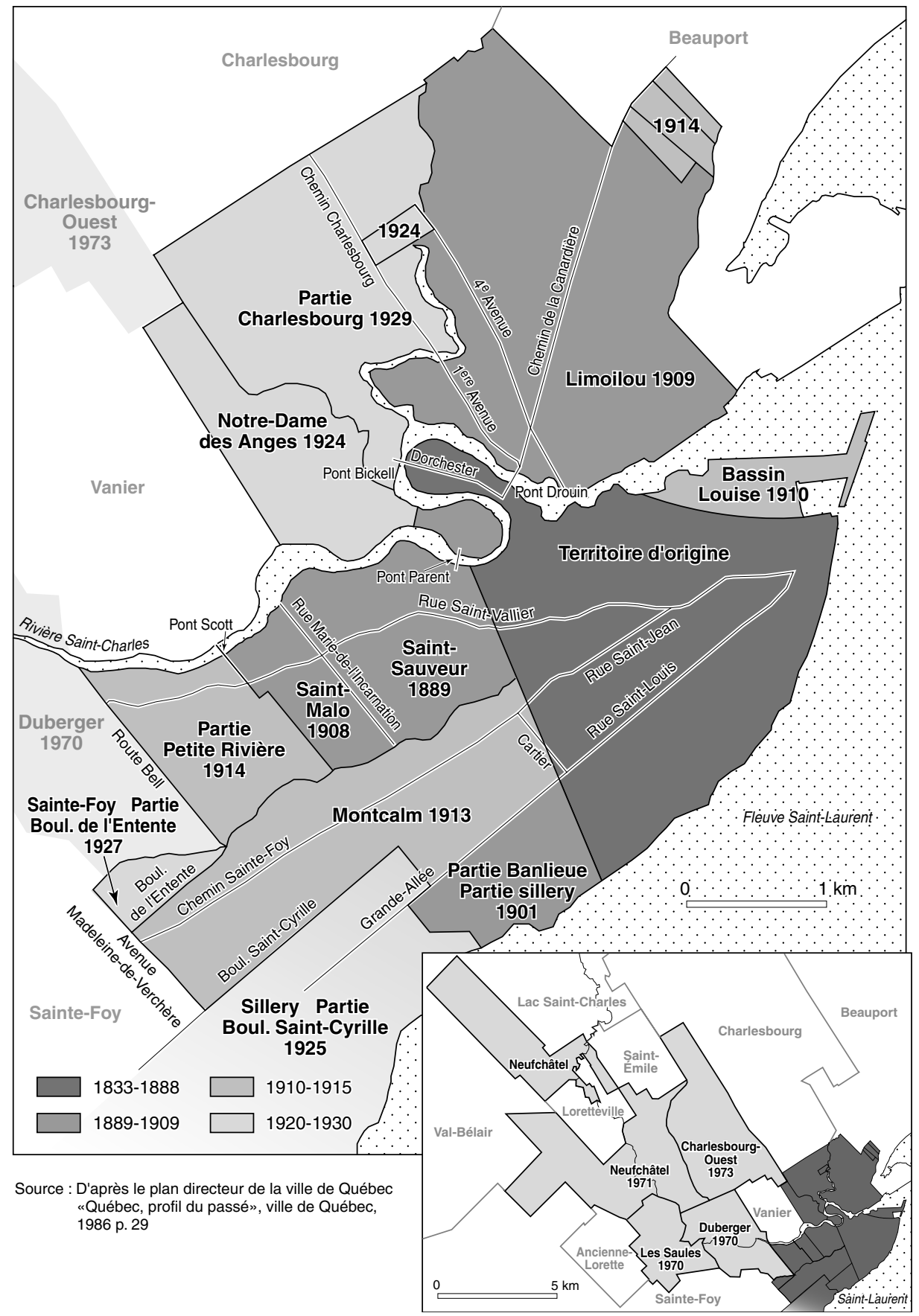




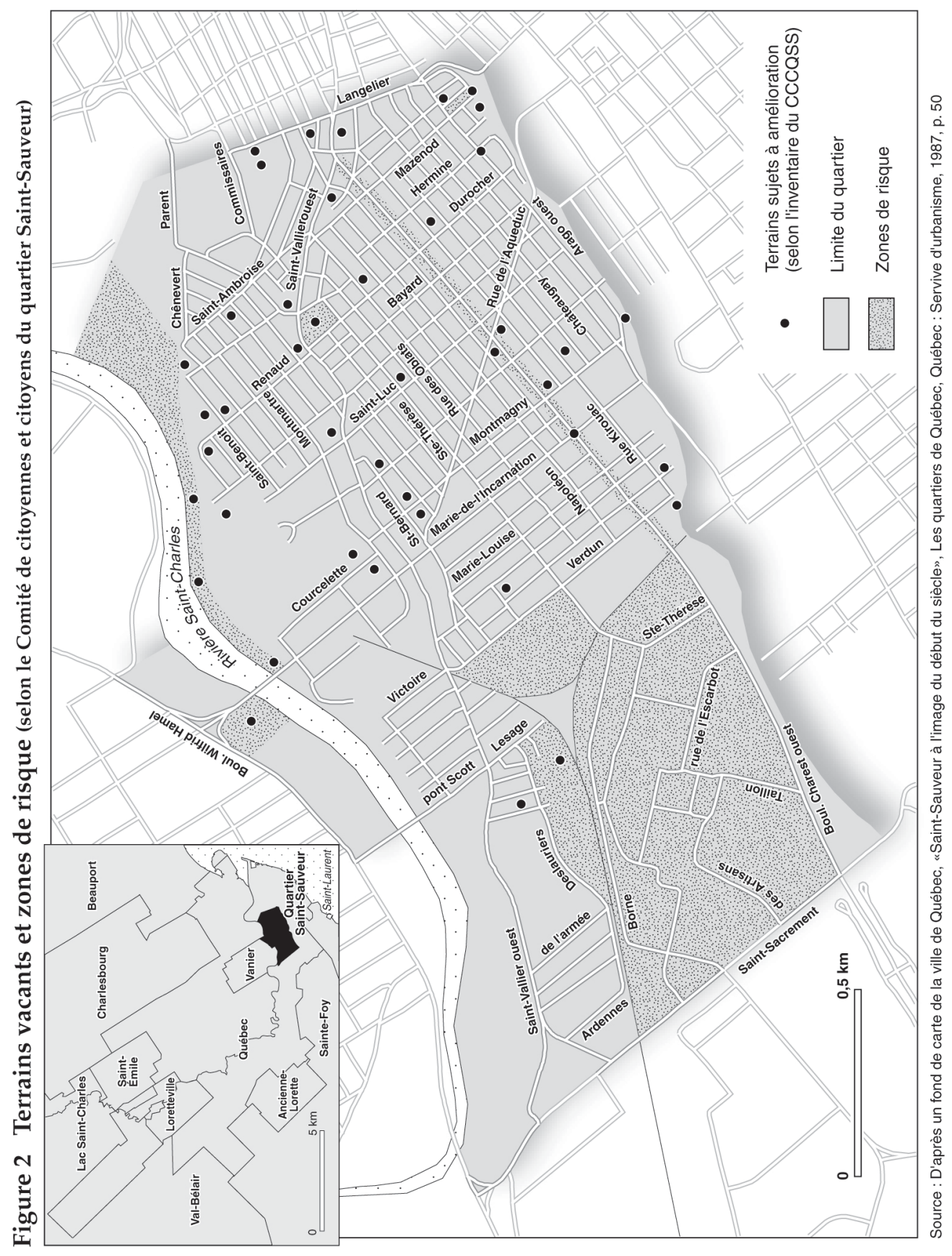


En réponse, la Ville de Québec mettait en place quelques années plus tard un programme de dégagement de terrain concernant les maisons placardées ou abandonnées (Ville de Québec, 1978). Mais le programme n'a pas rempli ses objectifs, car à la suite de nouveaux incendies dans les années quatre-vingt, le Comité de citoyens et de citoyennes convoquait une nouvelle fois la presse pour reprocher à la Ville de Québec sa passivité dans l'application des règlements municipaux traitant de la démolition des bâtiments vétustes ${ }^{17}$.

Aussi aujourd'hui, à l'échelle du quartier, identifier le risque d'incendie permet-il de mettre en évidence les lieux vulnérables du quartier, lieux de conflits entre les différents usages urbains, autrement dit, de conflits de localisation. Il est possible de distinguer trois catégories, comme le suggère l'étude de Gilles Côté sur les conflits de localisation de la Ville de Québec (Côté, 1991). Le premier cas de figure concerne les incendies d'origine criminelle : l'incendie a été allumé avec l'intention de transformer l'affectation du bâtiment, par exemple. Dans le deuxième cas de figure, les habitants exigent la démolition de maisons jugées dangereuses et, devant l'inertie des autorités municipales, placardent eux-mêmes les maisons menaçantes. Troisième possibilité, les incendies éclatent souvent dans les lieux qui abritent des personnes socialement marginalisées, comme les maisons de chambres, par exemple. Les fausses alarmes proviennent en effet souvent des mêmes endroits. Ces derniers sont par ailleurs des lieux de conflits sociaux, comme c'est le cas des grands HLM comme Place de la Rive ou des logements sociaux plus petits comme rue Chateauguay et boulevard Charest Ouest. Dans cette perspective, la possibilité d'incendie peut aussi être considérée comme un risque dit social (catastrophe provoquée par la délinquance) s'il est provoqué volontairement, et non uniquement comme un risque technologique majeur ou un risque naturel.

À l'échelle de la municipalité de Québec, l'incendie est également un risque et semble là aussi être la traduction d'un conflit de localisation. Il apparaît en effet que, lorsqu'un incendie se déclare dans le quartier Saint-Sauveur, la catastrophe est nommément localisée par la presse, par les autorités ou encore par la police, alors que les incendies qui surviennent dans d'autres quartiers de la Ville de Québec ne sont pas nécessairement identifiés en fonction du secteur où ils se produisent, comme le fait ressortir la base de données sur les conflits de localisation. Pour le conseiller municipal du quartier Saint-Sauveur interrogé à l'époque de notre enquête, l'incendie est l'occasion de montrer le quartier du doigt et de lui bâtir une mauvaise réputation. La menace d'incendie semble dès lors être un enjeu dans les relations entre les différents quartiers de la Ville de Québec.

Ainsi, l'incendie est un acteur très présent dans l'histoire du quartier SaintSauveur, du moins, c'est ce qu'il est possible de dégager de la présentation de ces deux exemples. De plus, on n'y discute pas que cendres et débris ou reconstruction systématique post-catastrophe, mais politique, annexion, rentrées fiscales et modernisation. Examinons alors comment ces arguments s'articulent dans les différents discours et en quoi ils ont joué un rôle important dans la constitution et la transformation du quartier, autant matérielle qu'idéelle. 


\section{DISCUSSION SUR LE RÔLE DU RISQUE D'INCENDIE DANS LA TRANSFORMATION URBAINE}

On entrevoit déjà le rôle important du risque d'incendie dans l'espace urbain. Mais théoriquement, la place occupée par le risque reste à clarifier. En effet, les théories sur le risque ne confèrent pas de rôle particulier à celui-ci : il est majoritairement considéré comme un élément externe à la dynamique urbaine. Parmi les exceptions, il est possible d'évoquer les travaux d'André Dauphiné (2001), par exemple, qui mettent en relation les risques et la théorie des catastrophes de René Thom. Or, le rôle du risque qui se dessine dans l'exemple du quartier SaintSauveur et ce, à deux époques différentes, donne à penser que celui-ci est plutôt étroitement lié à la dynamique urbaine et aux acteurs qui la façonnent.

Un indice de cette liaison est le fait que la maîtrise de l'incendie n'est jamais totale. Des feux continuent d'éclater dans le quartier. En examinant leur localisation et les objets urbains qu'ils affectent, il ressort que les lieux de prédilection des incendies sont souvent des zones urbaines instables et des lieux identifiés comme dangereux par les différents acteurs. Le risque semble donc caractériser des espaces dont le changement de vocation est souhaité. Ces espaces se situent souvent aux marges du quartier. Les études de géoarchitecture réalisées par Luc Noppen et Lucie K. Morisset (2000) parviennent au même constat: les endroits à valoriser dans le quartier se situent souvent aux confins de celui-ci ou dans des lieux dont l'affectation et l'usage est peu lisible.

Dès lors, comment traiter théoriquement ce risque qui semble constitutif de la dynamique urbaine? Il ne peut en effet plus être considéré comme une succession d'erreurs/occasions survenant par épisodes dans l'évolution urbaine.

Une possibilité est de considérer l'incendie et sa menace comme faisant partie intégrante du processus de construction-reconstruction urbaine. S'ils sont compris comme instrinsèquement liés au processus de formation urbaine, cela suppose par conséquent de repérer et de prendre en compte la manière dont les acteurs définissent et associent les différents éléments qui composent ces transformations. Il faut donc se pencher un instant sur les modalités d'action, ici celles qui sont liées au risque d'incendie.

Pour ce faire, un cadre théorique pertinent pour l'analyse de la relation risqueterritoire est celui que propose la sociologie des sciences et des techniques. Ce cadrage théorique, éprouvé lors de recherches précédentes, permet un éclairage intéressant sur la place du risque dans les transformations urbaines. Au risque de paraître extrêmement schématique, nous nous bornerons ici à expliciter seulement les éléments théoriques permettant cette compréhension ${ }^{18}$.

Ce courant de pensée porte toute son attention sur la façon dont les acteurs définissent et associent les différents éléments qui composent leur action. Sa principale méthodologie consiste à repérer et à prendre en compte ces différentes associations. Par conséquent, cette façon de considérer le rôle des acteurs, et par là même l'action en tant que telle, suppose de suivre les chaînes d'interaction composant les entités. Pour cela, indiquent Michel Callon et John Law (1997 : 109 110), « il suffit de suivre le procès de leur configuration et de leur (éventuelle) 
stabilisation. Au lieu de considérer les entités comme des réalités séparées les unes des autres, éventuellement coordonnées par des règles ou des conventions, il faut les voir comme représentant des réseaux d'éléments hétérogènes, chaque élément étant à nouveau un réseau ».

La définition de l'entité comme un réseau associant des éléments hétérogènes conduit les deux auteurs à mettre en évidence la double dimension de l'action : elle se prolonge toujours et se transmet continuellement. "Toute entité est à géométrie variable car elle se transforme en même temps que se modifie la configuration des actions qu'elle prolonge et qu'elle transmet » (Callon et Law, 1997 : 115).

Cependant, dans ce mouvement de débordement-prolongement, des résistances peuvent se produire. L'entité ne se conforme pas toujours aux prévisions ${ }^{19}$. Cela semble être le cas pour le risque d'incendie, qui a tendance à surgir là où on ne l'attend pas nécessairement. Dans l'exemple du quartier Saint-Sauveur, nous avons pu constater que le programme de dégagement de terrain mis en place par la Ville de Québec dès 1978 en réponse à l'inventaire des maisons abandonnées réalisé par le Comité de citoyens et de citoyennes n'a pas éliminé le risque d'incendie du quartier. Il en est de même lorsque le même acteur a décidé l'installation de gicleurs automatiques dans les maisons de chambres, par exemple, ou de détecteurs de fumée dans tous les logements. Le risque d'incendie a diminué, mais l'incendie continue de se manifester ailleurs, sous d'autres formes inédites. D'une certaine façon, le risque résiste à l'action entreprise pour l'éliminer.

Pour pouvoir rendre compte de la « circulation » de ces entités, c'est la notion de traduction qui permet d'affiner la compréhension de ce processus. La traduction, précise Dominique Vinck, « est un processus général par lequel le monde social et naturel se met progressivement en forme et se stabilise. Des éléments de ces socionatures sont déplacés et ces déplacements présentent une relative permanence. La traduction comprend plusieurs étapes se chevauchant éventuellement : la problématisation, l'intéressement, l'enrôlement et la mobilisation d'alliés » (Vinck, 1995 : 201). En résumé, traduire, c'est déplacer. Mais pour Michel Callon, traduire, $c^{\prime}$ est aussi exprimer dans son propre langage ce que les autres disent et veulent. $C^{\prime}$ est s'ériger en porte-parole : «La traduction n'est rien d'autre que le mécanisme par lequel un monde social et naturel se met progressivement en forme et se stabilise » (Callon, 1986 : 205).

Le même auteur explique dans un article sur le travail de la conception en architecture que "pour faire apparaitre les traductions il [...] suffit de suivre les acteurs eux-mêmes car ceux-ci se posent précisément le même problème que l'analyste : comment faire tenir ensemble [...] des intérêts économiques, l'action sociale et la forme des bâtiments sans pour autant tomber sous la coupe des ingénieurs? » (Callon, 1996 : 34).

Dans le cas du risque d'incendie, ne serions-nous pas en présence d'une " entité » composée d'éléments hétérogènes qui fait l'objet d'une série de traductions et de recomposition, pour reprendre la terminologie de la sociologie des sciences et techniques? En effet, comme l'ont montré Michel Callon et Arie Rip (1992 : 154), le risque participe à de longues chaînes d'interactions entre les artefacts techniques, 
les substances naturelles, les êtres humains organisés ou désorganisés. Cette approche théorique suppose ainsi de se distancer d'une vision uniquement socioculturelle du risque pour l'inscrire dans une perspective sociotechnique.

L'analyse des discours relatifs à la menace d'incendie permet de mettre en évidence l'étroite relation des risques avec des enjeux plus généraux quant au devenir de la Ville de Québec et de ses quartiers. Il semblerait, d'une part, que la question du risque ait joué un rôle important dans les discussions liées à la modernisation (annexion ou autonomie politique notamment). D'autre part, la prise en compte du risque d'incendie a déclenché une série d'actions urbaines qui ont profondément modifié le visage du quartier ainsi que sa relation avec les autres quartiers.

\section{UN DILEMME : AUTONOMIE OU MODERNISATION?}

À la lumière des éléments historiques vus précédemment, il ressort que SaintSauveur était un territoire convoité par la Ville de Québec. Cette dernière, d'après Lemoine (1982), voulait agrandir son territoire et augmenter le nombre de ses contribuables. Le risque d'incendie sert d'argument majeur dans la discussion. Or Saint-Sauveur résiste. Elle fait même l'affront à la Ville de Québec de devenir indépendante pendant douze ans. C'est paradoxalement le risque qui changera la situation en se transformant en catastrophe : l'incendie de 1889 révèle l'extrême urgence de trouver une solution et met ainsi un terme à la discussion sur l'annexion. L'argument de la Ville de Québec est d'ordre financier (obtenir plus de contribuables pour rentabiliser le nouvel aqueduc) et d'ordre politique (pouvoir se mesurer aux autres grandes villes d'Amérique du Nord qui, à cette époque-là, sont toutes engagées dans un vaste mouvement d'annexion de leurs banlieues). À SaintSauveur, les opinions se partagent entre les partisans du progrès et de l'annexion, qui désirent améliorer la qualité de vie des citoyens, et les tenants du statu quo, qui, à coup de slogans anti-taxes et anti-impôts, se regroupent en un parti politique appelé « anti-progressiste ».

Au cours des années 1990, quels sont les discours énoncés autour de la menace d'incendie? Les résidants du quartier associent fortement la dégradation d'un bâtiment à la possibilité qu'un incendie y survienne. Ils ont peur d'habiter à proximité de maisons de chambres, logements souvent plus vétustes. Certains vont même chercher à éviter de résider à côté de celles-ci. Dans cette perspective, le Comité de citoyens a réalisé un inventaire de maisons abandonnées, placardées ou jugées dangereuses. De son côté, la Ville de Québec assure dans son plan directeur de 1988 qu'elle a la maîtrise du feu et que, si Saint-Sauveur est « par nature » plus encline aux incendies à cause de la trame urbaine serrée qui la caractérise, le nombre de sinistres a nettement diminué. La Ville s'engage dans ce document à diminuer encore davantage le risque d'incendie, en mettant sur pied des programmes de subvention pour installer des gicleurs automatiques dans les maisons de chambres, par exemple, ou des détecteurs de fumée dans tous les logements. Pourquoi tous ces efforts alors que la situation s'est déjà améliorée? Parce que la menace d'incendie est un obstacle possible au mouvement désiré par la Ville de Québec, qui entend favoriser, et même provoquer, un retour de la population au centre-ville. 
Le discours de la Ville de Québec dans le plan directeur de 1988 rappelle étonnamment celui de la fin du XIX siècle. Il est intéressant de noter que déjà un siècle auparavant, la Ville de Québec avait le même désir (augmenter le nombre de contribuables, afin de pouvoir combler le déficit causé par la construction de l'aqueduc municipal). Cela signale peut-être une certaine récurrence dans la dynamique urbaine de Québec.

Au troisième niveau, celui de la municipalité, la possibilité d'incendie dans le quartier Saint-Sauveur peut, d'une part, engendrer un exode de la population résidante du quartier et, d'autre part, dissuader d'autres personnes de s'y établir. Le quartier peut se vider de ses habitants. Enfin, ceux qui restent sont probablement sans capacité financière, ce qui dans la perspective de la Ville de Québec ne semble pas rejoindre ses objectifs de manière suffisante, tant au XIX ${ }^{\mathrm{e}}$ qu'au $^{\prime} \mathrm{XX}^{\mathrm{e}}$ siècle. En effet, l'annexion se produit durant une période de stagnation de la population de Québec et vise directement à augmenter les effectifs de la Ville.

Le discours sur l'incendie semble dans cette perspective être orienté pour combattre un risque majeur aux yeux de la Ville de Québec : la stagnation de la population. Pourtant, le problème reste d'actualité, vu qu'une augmentation ne s'est pas produite (de fait, le dernier recensement enregistre encore une baisse). Ainsi, il apparaît que le discours sur le risque d'incendie s'appuie sur différentes peurs et s'en sert pour justifier son action et ses ambitions (annexion, modernisation du quartier, etc.).

\section{INNOVATION URBAINE ET UNIFORMISATION}

Il est possible de faire ressortir un second rôle du risque dans le territoire, lié à l'innovation urbaine et à l'uniformisation. En effet, cet exemple met en évidence combien la potentialité d'un sinistre (le risque) peut être reliée à des actions urbaines. Plus précisément encore, on s'aperçoit que le risque, une fois qu'il est identifié et nommé dans des discours, motive directement la réalisation d'une ou de plusieurs actions en milieu urbain. C'est le cas par exemple de la transformation du boulevard Langelier en un espace coupe-feu d'avec le quartier Saint-Roch ou de l'annexion du quartier Saint-Sauveur qui permet la modernisation totale du quartier aux frais de la municipalité de Québec. Il est possible à cet égard de souligner trois éléments :

Premièrement, on remarque que l'incendie donne la possibilité, sinon d'améliorer ce qui existe dans l'organisation urbaine, du moins de le modifier. En effet, la catastrophe suscite des actions en vue de prévenir une nouvelle catastrophe. Le nouveau cadre bâti, c'est-à-dire les reconstructions et les nouvelles constructions, va être modifié en tenant compte de la possibilité d'une catastrophe, c'est-à-dire du risque. Ainsi, non seulement la catastrophe modifie la ville, puisqu' elle concourt à perturber et à transformer le cadre bâti existant ainsi que les structures urbaines, mais encore le risque lui-même contribue à modifier l'espace urbain du fait qu'il finit par être intégré aux actions de prévention - sous forme notamment de mesures de sécurité, de nouveaux règlements, etc.

Le risque et la catastrophe apparaissent alors intimement imbriqués dans la dynamique urbaine : ils participent à la dynamique de construction-reconstruction, ils en sont les moteurs et, même, les éléments innovateurs. Par exemple, les 
premières mesures de sécurité prises au XVIII ${ }^{e}$ siècle contre le danger d'incendie contribuent à forger un nouveau style architectural. En effet, selon Noppen, la pénurie de terrains à bâtir et les risques de plus en plus grands d'incendie obligent les autorités à émettre des règlements plus précis pour régir la construction en ville. L'Ordonnance de 1727 préparée par l'ingénieur de Léry et promulguée par l'intendant Dupuy interdit de construire en bois dans les villes, fixe le nombre d'étages à deux, défend d'ériger des toits mansardés, conseille sur le matériau à utiliser pour la toiture, $l^{\prime}$ isolement des cheminées, proscrit toute boiserie apparente à l'extérieur des maisons. Pour Noppen, cette Ordonnance souligne deux éléments intéressants. Tout d'abord, on passe de la protection individuelle des maisons à celle d'un ensemble urbain : «En effet, au XVII siècle, les couvertures en ardoise, les escaliers extérieurs, la distance qui séparait chaque bâtiment et les échelles disposées près des cheminées étaient des précautions qui visaient à protéger chaque maison et ses habitants. L'Ordonnance de 1727 cherche plutôt à protéger l'ensemble urbain, au détriment de l'apparence et du caractère individuel de chaque habitation » (Noppen, 1989 : 24).

De plus, l'Ordonnance entraîne des innovations architecturales qui s'insèrent dans le milieu urbain : « les murs coupe-feu, l'implantation de cheminées dans ces murs ou dans les pignons, la charpente à filières (ou à pannes) et la couverture en fer-blanc sont autant d'innovations qui surviennent au bon moment» (Noppen, 1989 : 25). À cet égard, l'aménagement du boulevard Langelier en 1885, élargi d'un terre-plein planté d'arbres au centre, apparaît bien comme une innovation qui s'est imposée à la suite de la prise en compte du risque d'incendie. Sa vocation strictement utilitaire de coupe-feu n'en constitue pas moins une nouveauté dans le paysage urbain de la ville de Québec de l'époque. Ainsi, l'identification du risque conduit à, voire encourage, des formes d'innovation urbaine.

Deuxièmement, il est intéressant de remarquer que la valeur économique d'un objet ou d'une situation identifiée comme « à risque » ne semble pas être le critère décisif pour déclencher une politique de gestion du risque. Ce sont bien plus les conséquences futures espérées qui motivent une décision d'action, où domine l'espoir de récolter les gains (de toutes sortes, pas uniquement en monnaie) de l'opération. En ce sens, l'annexion de Saint-Sauveur est véritablement une prise de risque pour la Ville de Québec, au sens fort du terme. Celle-ci espère obtenir une plus-value importante de l'opération d'annexion, dont elle estime le coût à 406000 \$ en 1889 (Lemoine, 1982). Malgré la précarité et les mauvaises conditions socio-économiques régnant dans cette portion de territoire, la Ville de Québec est prête à investir des sommes importantes pour annexer la municipalité, dans le but d'agrandir le territoire de la Ville, le nombre de contribuables et le prestige qui va de pair. Malheureusement, l'annexion ne semble pas avoir eu l'effet escompté : aujourd'hui encore, la municipalité de Québec recherche des contribuables capables de payer des impôts.

Troisièmement, simultanément au caractère innovateur qui découle du risque, il semblerait également que sa prise en compte favorise une certaine uniformisation du paysage architectural. Par exemple, à la suite de l'Ordonnance de 1727, on assisterait dans la Basse-Ville de l'époque à la disparition d'ornements - attributs stylistiques - qui réduisent la maison à sa forme essentielle, c'est-à-dire au type architectural (Noppen, 1989 : 24). Dans le cas de Saint-Sauveur, l'uniformisation 
que l'on constate vient avec la modernisation du quartier entreprise à la suite de l'annexion de ce dernier par la Ville de Québec : les trottoirs, les égouts, les chaussées en pierre, contribuent à moderniser le quartier, certes, mais aussi à le faire ressembler au reste de la ville. À l'uniformiser. Les signes mêmes du progrès s'identifient par leur ressemblance avec ceux de la Haute-Ville. Aussi le risque motive-t-il l'uniformisation, en même temps qu'il engendre des formes d'innovation urbaine.

\section{CONCLUSION}

Ce dernier point nous permet d'élargir la perspective dans laquelle le rôle du risque dans le quartier peut être pensé. Il est ainsi possible, en guise de conclusion, de prolonger l'analyse du risque d'incendie dans le quartier Saint-Sauveur effectuée à deux périodes distinctes en faisant ressortir trois points particulièrement intéressants.

Premièrement, il apparaît que le risque d'incendie peut être lu comme une entité hétérogène. Parfois classé sous la catégorie "risque social » lorsqu'il s'agit d'un incendie issu d'un immeuble vétuste, il peut également être classé comme « risque naturel » dans le cas de feux de forêts, ou encore de « risque environnemental » et « technologique » lorsqu'il éclate à la suite d'une émanation toxique industrielle, par exemple. Est-il encore pertinent de distinguer, dans l'analyse, ces différents visages du risque d'incendie? Sur un plan théorique, cette perspective nous conduit davantage à considérer le risque d'incendie comme un élément " générique ", dont les manifestations peuvent être hétérogènes. En effet, malgré la variété de ses manifestations, la "personnalité » de l'incendie et de sa menace demeure permanente dans le quartier et ce, dans la durée, puisqu'il s'inscrit en continu dans l'historiographie du quartier Saint-Sauveur.

Deuxièmement, le risque d'incendie résiste aux politiques d'actions urbaines mises en place. Certes, il réagit aux mesures de prévention, mais cela ne l'empêche pas de se manifester à d'autres endroits, plus fragiles, plus vulnérables, souvent aux marges du quartier. En analysant cette résistance à l'action entreprise pour le faire disparaître du quartier, il est possible de mettre en évidence les lignes de fracture et de vulnérabilité du quartier. En ce sens, le risque contribue à délimiter ce dernier; dans notre cas spécifique, celui du risque d'incendie, il joue un rôle actif dans le processus de configuration/reconfiguration du quartier. En d'autres mots, il apparaît que le risque d'incendie a bien contribué à créer le quartier Saint-Sauveur tant matériellement que symboliquement, en demeurant un élément du dispositif urbain significatif d'une identité mise à mal par la récurrence d'incendie dans le quartier. Selon cette optique, la création matérielle du quartier et la dimension idéelle et identitaire ne peuvent plus être lues comme des éléments séparés du dispositif : elles font partie du même processus de configuration/reconfiguration, qui peut être analysé selon des termes similaires.

Cependant, la permanence et la récurrence du risque d'incendie dans le quartier Saint-Sauveur ne conduit pas uniquement à mettre en évidence les lieux plus vulnérables du quartier. Cette observation permet aussi de souligner, troisièmement, à quel point le risque fait constamment l'objet d'alliances et de traduction au cours du processus de formation urbaine. Il nous a été donné d'en entrevoir quelques aspects à travers l'analyse du rôle du risque d'incendie dans le 
quartier Saint-Sauveur. C'est notamment lorsque le risque se transforme en catastrophe que la discussion sur l'annexion se clôt. Les tenants de l'annexion se sont alliés au risque. Il en est de même avec la prise en compte du risque dans le nouveau cadre bâti : il faut l'ingénieur de Léry, l'intendant Dupuy, un nombre d'étages, une Ordonnance : autant d'éléments différents qui traduisent le risque et qui contribuent à solidifier et à concrétiser son action dans le dispositif. Désormais, $c^{\prime}$ est toute une architecture qui ressort modifiée, la prise en compte du risque d'incendie ayant entraîné des modifications importantes, tant sur le plan des matériaux que de l'organisation spatiale du bâti. D'une certaine manière, enfin, c'est tout un territoire, au sens géographique du terme, qui en ressort transformé.

Finalement, nous avons vu que malgré la diversité des époques historiques, le risque d'incendie connote de manière permanente le quartier Saint-Sauveur. Il apparaît que la présence du risque d'incendie n'a plus rien de « naturel » dans le quartier Saint-Sauveur : il n'est pas uniquement lié à la densité du tissu urbain, ni simplement lié aux conditions socio-économiques du quartier. Son rôle est davantage celui d'un acteur parmi d'autres, dont la portée est capable de modifier durablement un espace urbain et d'y laisser son empreinte. En ce sens, il est possible d'affirmer que le risque d'incendie contribue à structurer le territoire analysé.

\section{NOTES}

1 Mes remerciements vont à Guy Mercier et à Ruggero Crivelli qui ont bien voulu relire et commenter une version antérieure du manuscrit. Pour la version définitive de ce texte, je suis fortement redevable aux trois lecteurs anonymes pour leurs commentaires éclairants. J'aimerais aussi remercier les Cahiers de géographie du Québec pour leur travail d'infographie et de traduction.

2 Se reporter par exemple au numéro des Annales de Géographie (2002) no 627-628 portant sur les approches géographiques des risques «naturels » sous la direction de Patrick Pigeon.

3 Voir par exemple les travaux de Susan Cutter (1994 et 1996 notamment).

4 Pour une bonne idée des réflexions en cours, voire notamment la série de publications issues du Programme risques collectifs et situations de crise (1994-2001), CNRS, ainsi que les Actes du colloque international «Risques et territoires », mai 2001, Lyon, ENTPE/ CNRS, 3 volumes.

5 Un numéro de la Revue de géographie alpine a été consacré à cette thématique : « risques naturels et croissance urbaine » $\left(n^{\circ} 4,1994\right)$. Par exemple, les travaux de Robert D’Ercole sur l'éruption volcanique du Cotopaxi et la ville de Quito (1994 notamment).

6 Se reporter par exemple au numéro spécial des Annales de la Recherche Urbaine intitulé " risques et périls" ( $\left.n^{\circ} 40,1988\right)$.

7 Pour plus de détails sur ces deux notions, voir November 2002.

8 L'espérance de vie de la population de la Basse-Ville était de huit ans inférieure à celle de la région, selon le Profil de la population et des ressources sociosanitaires de la région de Québec (1992: 15).

9 Il faut mentionner à cet égard la très récente " renaissance » du quartier Saint-Roch, quartier qui jouxte à l'est le quartier Saint-Sauveur, grâce à une intervention urbaine prioritaire et massive des pouvoirs publics (Mercier, 1998). 
10 Notamment, en plus des études du Secrétariat aux Affaires régionales, le Rapport de la commission sur le maintien des institutions et les mesures de repeuplement des quartiers centraux de Québec (1991), Conseil des affaires sociales, CEQ et Ville de Québec; le Diagnostic socio-économique des quartiers du centre de Québec (1992), Comité pour la relance de l'économie et de l'emploi du centre de Québec (CREECQ); et Stratégie d'intervention pour le développement économique et communautaire des quartiers centraux de Québec 19931998 (1993), CREECQ, SQDM - Québec.

11 Cette étude a été réalisée dans le cadre d'une maîtrise effectuée au département de géographie de l’Université Laval durant l'année 1993. Elle comportait une partie théorique et une étude de cas. La recherche de terrain a porté sur le quartier SaintSauveur dans lequel il s'agissait de mettre en relation plusieurs catégories de risques (industriels, technologiques, naturels, sociaux ou encore environnementaux) sur un même territoire.

12 Les deux moments-clés qui illustrent la relation du risque d'incendie avec la dynamique $\mathrm{du}$ territoire servent avant tout à stimuler la discussion. Nous aurions pu aborder d'autres séquences temporelles, mais le manque de documentation sur le quartier Saint-Sauveur a constitué un frein à l'analyse d'autres événements.

13 Par exemple, le Bureau régional de Québec du Secrétariat aux Affaires régionales, ou encore la Direction régionale de Québec de l'Office de planification et de développement du Québec.

14 L'enquête portait sur plusieurs catégories de risques, rappelons-le. Nous ne rapportons ici que le risque d'incendie.

15 Les maisons de chambres, selon l'appellation québécoise, sont des maisons qui ne contiennent que des chambres individuelles à louer.

16 Ce sont les termes utilisés par les personnes interrogées : il s'agit de maisons dont l'accès est interdit pour raisons de sécurité, au moyen de planches ou d'un mur de briques.

17 G. Dubé, Quartier Saint-Sauveur, des citoyens exigent la démolition de trois maisons " dangereuses ", Le Soleil, 14 février 1980, p. A3.

18 Il s'agit ici d'une présentation tout à fait sommaire de quelques concepts élaborés par la sociologie des sciences et techniques. Le lecteur curieux se rapportera aux ouvrages de Jasanoff et al., 1994 pour une vue d'ensemble de cette approche, ainsi qu'aux nombreux travaux de B. Latour (notamment 1999 et 2001). Pour une discussion plus approfondie sur l'apport de ces concepts à une réflexion géographique, voir November, 2002.

19 Michel Callon et John Law ont analysé un exemple de production de résistance dans le cadre du projet d'avion supersonique britannique : « l'avion (TSR2) résistait (les moteurs explosèrent, les difficultés s'accumulèrent, l'avion refusait de se plier aux projets concoctés par les militaires) et, en résistant, il modifiait la distribution des entités associées, leurs visées. La définition de ce qui est réalisable et de ce qui ne l'est pas se décide dans l'interaction. Il n'est pas possible, avant d'en avoir éprouvé le réalisme, de savoir comment les entités vont se comporter, et cela est encore plus vrai lorsqu'il s'agit de science et de technique. Qui pouvait dire comment le projet d'un avion décollant verticalement allait évoluer? Quelle forme il allait prendre? S'il allait résister, se comporter comme un véritable agent et en retour modifier les actions en cours? Ou, au contraire, se plier docilement aux projets et se réduire à un objet passif, simplement. Le TSR2 ne se contente pas de prolonger les actions qui le mettent en forme; il les relance dans des directions qui sont parfois imprévisibles » (Callon et Law, 1997 : 105). 


\section{BIBLIOGRAPHIE}

BÉLANGER, Hervé (1993) Les zones défavorisées de la CUQ, un éclairage historique. Office de Planification et de Développement du Québec, direction régionale de Québec.

BLANCHER, Philippe et LAVIGNE, Jean-Claude (1991) Risques et réseaux urbains, génie urbain: un état des connaissances et des savoirs-faire. Lyon, Économie et Humanisme, collaboration INGUL.

CALLON, Michel (1986) Eléments pour une sociologie de la traduction : la domestication des coquilles Saint-Jacques et des marins pêcheurs dans la baie de Saint-Brieuc. L'Année sociologique, (36) : 169-206.

(1992) Sociologie des sciences et économie du changement technique : l'irrésistible montée des réseaux technico-économiques. Dans collectif CSI (éd.) Ces réseaux que la raison ignore. Paris, L'Harmattan, pp. 53-78.

(1996) Le travail de la conception en architecture. Dans D. Valabrège (dir.) Situations, Les Cahiers de la Recherche Architecturale, (37) : 25-35.

CALLON, Michel et RIP, Arie (1992) Humains, non-humains : morale d'une coexistence. Dans J. Theys et B. Kalaora (dir.) La Terre Outragée, les experts sont formels. Paris, Éd. Autrement, (1) : 140-156.

CALLON, Michel et LAW, John (1997) L'irruption des non-humains dans les sciences humaines. Dans B. Reynaud (dir.) Les limites de la rationalité, t.2 Les figures du collectif, Colloque de Cerisy. Paris, La Découverte, pp. 99-118.

CALLON, Michel, LASCOUMES, Pierre et BARTHE, Yannick (2001) Agir dans un monde incertain : essai sur la démocratie technique. Paris, Seuil.

CHALINE, Claude et DUBOIS-MAURY, Jocelyne (1994) La ville et ses dangers : prévention et gestion des risques naturels, sociaux et technologiques. Paris, Masson.

CHATEAURAYNAUD, Francis et TORNY, Didier (1999) Les sombres précurseurs : une sociologie pragmatique de l'alerte et du risque. Paris, Éditions de l'École des Hautes Études en Sciences Sociales.

CREECQ (Comité pour la relance de l'économie et de l'emploi du centre de Québec) (1993) Stratégie d'intervention pour le développement économique et communautaire des quartiers centraux de Québec 1993-1998. SQDM - Québec.

CÔTÉ, Gilles (1991) Conflits de localisation et luttes urbaines dans l'agglomération de Québec. Mémoire de fin de cycle, Département de géographie, Université Laval.

CUTTER, Susan, dir. (1994) Environmental Risks and Hazards. London, Prentice-Hall.

(1996) Les réactions des sociétés aux risques écologiques, Revue internationale des sciences sociales, (150) : 603-615.

DAUPHINÉ, André (2001) Risques et catastrophes : observer - spatialiser - comprendre - gérer. Paris, Armand Colin.

DECROP, Geneviève, DOURLENS, Chistine et VIDAL-NAQUET, Pierre et al. (1997) Les scènes locales de risque. Grenoble, Plan Urbain.

DELUMEAU, Jean et LEQUIN, Yves (1987) Les malheurs des temps : histoire des fléaux et des calamités en France. Paris, Larousse.

D'ERCOLE, Robert (1994) Mesurer le risque: le volcan Cotopaxi et les populations proches. Dans CHAM'S, Enseigner les risques naturels : pour une géographie physique revisitée, Montpellier/Paris, GIP Reclus, Anthropos, pp. 111-150. 
DIEUDONNÉ, Patrick, dir. (1994) Villes reconstruites. Du dessin au destin. Paris, L'Harmattan.

DONNELL, Robert Phippen (1980) Fire in the City: Spatial Perspectives on Urban Structural Fire Problems. New York, Syracuse University, PhD thesis.

DROUIN, Marie-Christine (1988) La rénovation domiciliaire dans Saint-Sauveur : nécessité et bilan. Québec, Université Laval, Département de géographie, mémoire de $1^{\mathrm{er}}$ cycle non publié.

DUBÉ, Guy (1980) Quartier Saint-Sauveur, des citoyens exigent la démolition de trois maisons «dangereuses ». Dans Le Soleil, 14 février, p. A3.

DUBOIS-MAURY, Jocelyne (1988a) Un risque urbain permanent: le feu. Annales de géographie, (539) : 84-95.

(1988b) La vulnérabilité de la ville à l'incendie. Les Annales de la recherche urbaine, (40) : 65-72.

DUBOIS-MAURY, Jocelyne et CHALINE, Claude (2002) Les risques urbains. Paris, Armand Colin.

GUERRIER, Philippe et PATRY, Robert (1987) Profil environnemental du territoire du département de santé communautaire de l'hôpital du Saint-Sacrement. Québec, Département de santé communautaire, Hôpital du Saint-Sacrement.

HARE, John, LAFRANCE, Marc et RUDDEL, David-Thiery (1987) Histoire de la Ville de Québec, 1608-1871. Montréal, Boréal/Musée canadien des civilisations.

JASANOFF, Sheila, MARKLE, Gerald, PETERSEN, James et PINCH, Trevor, dir. (1994) Handbook of Science et Technology Studies. London, Sage Publications.

KASPERSON, Jeanne X. et KASPERSON, Roger E., dir. (2001) Global Environmental Risk. London, Earthscan/The United Nations University Press.

LATOUR, Bruno (1999) Politiques de la nature : comment faire entrer les sciences en démocratie. Paris, La Découverte.

(2001) L'espoir de Pandore. Pour une version réaliste de l'activité scientifique. Paris, La Découverte.

LAVIGNE, Jean-Claude et al. (1988) Dynamique urbaine et gestion des risques : les processus en jeu dans la COURLY. Paris, Plan Urbain.

LEBEL, Alyne (1983) Les facteurs du développement urbain. Dans G. Dagneau (dir.) La Ville de Québec, histoire municipale IV : de la Confédération à la charte de 1929. Cahiers d'Histoire, no 35, Québec, La Société historique de Québec, pp. 31-47.

LEMOINE, Réjean (1982) La bataille des annexions : Saint-Sauveur 1889. Droit de parole, $9(9): 6-7$.

LUPTON, Deborah, dir. (1999) Risk and Sociocultural Theory: New Directions and Perspectives. Cambridge, Cambridge University Press.

LUSSIER, Jacques, dir.(1992) Diagnostic socio-économique des quartiers du centre de Québec. Québec, Comité pour la relance de l'économie et de l'emploi du centre de Québec (CREECQ).

MERCIER, Guy (1998) Le jardin ou la destinée humaine de la nature. Dans G. Mercier et J. Bethemont (dir.) La ville en quête de nature. Québec, Septentrion, pp. 7-13.

MORISSET, Lucie K. (2000) Histoire de la forme urbaine du quartier Saint-Sauveur. Québec, Ville de Québec, Centre de développement économique et urbain, Division du design urbain et du patrimoine. 
NOPPEN, Luc (1989) L'arrondissement historique du Vieux Québec. ARQ/Architecture Québec, no 51.

(2000) Architecture et identités. Histoire de l'architecture de Saint-Sauveur. Québec: Ville de Québec, Centre de développement économique et urbain, Division du design urbain et du patrimoine.

NOPPEN, Luc et MORISSET, Lucie K. (2000) Patrimoine du quartier Saint-Sauveur : rapport de synthèse. Québec, ville de Québec, Centre de développement économique et urbain, Division du design urbain et du patrimoine.

NOVEMBER, Valérie (1994) Rôle et nature du risque dans la structure urbaine : illustration par le quartier Saint-Sauveur, à Québec. Québec, Université Laval, Département de géographie, Faculté des études supérieures.

- (2002) Les territoires du risque : le risque comme objet de réflexion géographique. Berne, Peter Lang.

CRSS DE QUÉBEC (1992) Profil de la population et des ressources sociosanitaires de la région de Québec. Québec, DSC du CHUL, DSC de l'Hôpital de l'Enfant-Jésus, DSC de l'Hôpital du Saint-Sacrement.

CONSEIL DES AFFAIRES SOCIALES (1991) Rapport de la commission sur le maintien des institutions et les mesures de repeuplement des quartiers centraux de Québec. CEQ et Ville de Québec.

REQUÊTE DES CITOYENS DU QUARTIER SAINT-MALO (1972) « Faire disparaître ou remplacer la vieille usine de Québec-Gaz ». Dans Le Soleil, 25 mai.

VILLE DE QUÉBEC, Service d'urbanisme, division habitat (1978) Programme de dégagement de terrains.

VILLE DE QUÉBEC, Service d'urbanisme (1987) Saint-Sauveur à l'image du début du siècle, coll. Les quartiers de Québec. Québec.

(1988) Une ville sur mesure, Plan directeur d'aménagement et de développement de la Ville de Québec, Québec.

VINCK, Dominique (1995) Sociologie des sciences. Paris, Armand Colin. 